\title{
Repeated observation of an uncertain signal
}

\author{
JOHN A. SWETS \\ Bolt Beranek and Newman Inc., Cambridge, Massachusetts 02138 \\ and \\ THEODORE G. BIRDSALL \\ University of Michigan, Ann Arbor, Michigan 48104
}

\begin{abstract}
The focus here is on sensory adaptation, or progressively more appropriate attention, as repeated observations yield more information about a signal with an uncertain parameter. The signal was a brief sinusoid; its uncertain parameter was frequency. Detection performance is predicted from data on a signal of known and constant frequency, as a function of the number of frequencies the uncertain signal could assume. A comparison condition presented a signal that varied in a manner not permitting adaptation. Models derived from signal detection theory describe the ideal observation processes for the three signal conditions, and supply quantitative predictions of relative performances. The models are generally supported by the data.
\end{abstract}

We consider in this paper the process whereby repeated observations enhance the detectability of a signal with an uncertain parameter. This process is usually viewed as one of sensory adaptation. We examine here data obtained from human observers, and compare their performance to that expected of an ideal observer. The framework is the general theory of signal detectability (see Green \& Swets, 1966).

In our detection paradigm, a trial contains a fixed number of observation intervals, with independent samples of noise. A signal is presented in all or none of the observation intervals of a given trial. The observer responds "yes" (signal present) or "no" (signal absent) after each observation as a trial proceeds, taking into account the evidence from all previous observations of that trial. Combining many trials permits calculation of a detection index for each observation stage. The signal used in the experiment was a brief sinusoid; its uncertain parameter was frequency.

Three degrees of frequency uncertainty were studied. In the first case, there is nominally no frequency uncertainty: the signal frequency remains fixed throughout a group of trials. In the second case, there is frequency uncertainty that cannot be reduced by additional observations: the signal frequency varies randomly from one observation interval to

Joseph Markowitz participated in several phases of the design and execution of the experiment, and Kathleen Benson collected the data. We gratefully acknowledge their capable contributions. Collection of the data was supported by the Human Performance Branch of the NASA-Ames Research Center. This article was prepared during a sabbatical leave granted the first author. another within a trial. In the third case, initial frequency uncertainty can be overcome with time: the signal frequency varies randomly between trials but is fixed over the successive observation intervals of a given trial.

Drawing from analogous problems in testing statistical hypotheses, the signals of the first two cases may be termed "simple" signals, and the signal of the third case, a "composite" signal. We distinguish between the two types of simple signal by referring to the first of them as a "signal specified exactly" (SSE), reserving the term "simple" for the second type.

The next section describes a model whereby ideal performance with the composite signal may be predicted from performance with the signal specified exactly. The case of the composite signal, which clearly permits the observer to adapt, is the primary concern of this paper. Ideal models for predicting simple-signal performance from SSE performance, and for predicting the growth of SSE detectability over time, are also described. Both of these cases, of interest in their own right, serve here also as baseline conditions for the composite-signal case.

\section{MODELS}

This section describes various models for the growth of detectability over repeated observations of signals with and without an uncertain parameter. All of the models are based on a consideration of ideal performance, and all of them are based on the assumption that the normal detection index $\mathrm{d}^{\prime}$ is accurate enough as a description. One model defines the ideal growth of detectability over succes- 
sive observations, and is applied to the SSE and to the simple signal. Two models define the decrement in detectability resulting from frequency uncertainty, and may be applied, in different degrees, to both the simple and composite signals. Two other models specify the process by which information is stored and accumulated for the cases of the simple and composite signals, respectively.

\section{Signal Specified Exactly}

If the observer's decision variable is statistically independent from one observation to another, and if he combines observations over time with no loss of information, then his performance d' will increase as the square root of the number of observations $(\sqrt{n})$ no matter what the situation. The derivation of this prediction is given elsewhere (Green \& Swets, 1966, pp. 271-275).

The assumption of statistical independence for the SSE implies an observer so well trained on that signal frequency that no learning occurs as he makes repeated observations. Particularly, this assumption implies that the observer does not rezero in on the frequency each time he receives a reasonably good signal presentation.

\section{Simple Signal}

The ideal-observer model for the case of the simple signal also predicts that the performance index $\mathrm{d}^{\prime}$ will increase proportional to $\sqrt{n}$. By a procedure described later, the value of $d^{\prime}$ for the first observation under SSE is used to predict the value of $d^{\prime}$ on the first observation of a simple signal, and this latter value of $d^{\prime}$ is extended to succeeding observations by multiplying by $\sqrt{\mathrm{n}}$.

The ideal observer for the simple signal uses as many parallel filters as there are possible frequencies (M), with each filter tuned to one frequency. After the first observation, the largest filter output (on a log-likelihood-ratio scale) is stored and compared with a criterion for the "yes" response; on the second observation, the new values of the filter outputs for that observation alone are read, the maximum value is added to the previously obtained maximum value, and the sum is compared to a new criterion for the "yes" response; and so forth. This model derives from a basic theorem of detection theory, that log likelihood ratio is additive for independent observations.

\section{Composite Signal}

The ideal observer for the composite signal has been discussed by Nolte (1967). It xeeps the outputs of the $\mathbf{M}$ filters separated and stores them individually after each observation. The output of each filter on each observation is added to the cumulative output, obtained from previous observations, of the corresponding filter. After each observation, the largest of the $\mathbf{M}$ individual sums is compared to a criterion for the "yes" response. It is by this means, when the signal is present, that the output of the filter that passes the signal accumulates at a uniformly faster rate than the outputs of the other filters. It soon diverges from the others and gives a more reliable indication of signal presence than would be available if the outputs of the various filters were combined as they are by the ideal observer for the simple signal.

Though we shall not make use of the fact here, we should note that storing separately the outputs of the $\mathbf{M}$ filters provides a basis for signal identification as well as detection. In a unified process, the ideal observer for the composite signal accumulates not only information about signal likelihood, but also information abo $t$ which signal (if any) is present. Empirical evidence for the parallel growth of detection and identification is provided by Swets, Green, Getty, and Swets (1978).

In general, observations in the case of the composite signal are not statistically independent when the signal is present, and so the prediction of $\mathrm{d}^{\prime}$ proportional to $\sqrt{n}$ does not apply. We describe next a procedure whereby predictions of $\mathrm{d}^{\prime}$ for a composite signal at any given stage of observation are determined from the value of $d^{\prime}$ obtained under SSE at the same observation stage. That procedure may be used to predict the value of $d^{\prime}$ on the first observation of a simple signal, a case in which the prediction of $d^{\prime}$ proportional to $\sqrt{n}$ applies to observations after the first.

\section{One-of-M Orthogonal Signals}

To predict behavior in the cases of simple and composite signals from SSE behavior, we need a model for the decrement in detectability caused by frequency uncertainty. One such model is termed the model for "one-of-M orthogonal signals." This model shows what happens to ideal performancein terms of the relative, or receiver, operating characteristic (ROC curve)-as $M$ increases. It permits establishing the relationship between $\mathrm{d}^{\prime}$ for SSE and $\mathrm{d}^{\prime}$ for one of $\mathrm{M}$ frequencies.

Nolte and Jaarsma (1967) reported some fairly precise numerical solutions for the likelihood-ratio observer for many values of $M$. They found, incidentally, that the non-ideal observer that uses a common decision criterion for each of the $M$ parallel filters differs only infinitesimally from the ideal observer that uses a proper combination of the output of the filters. We therefore use this non-ideal observer, with little or no loss of computational accuracy, to derive ROC curves and other statistics for ideal performance. 


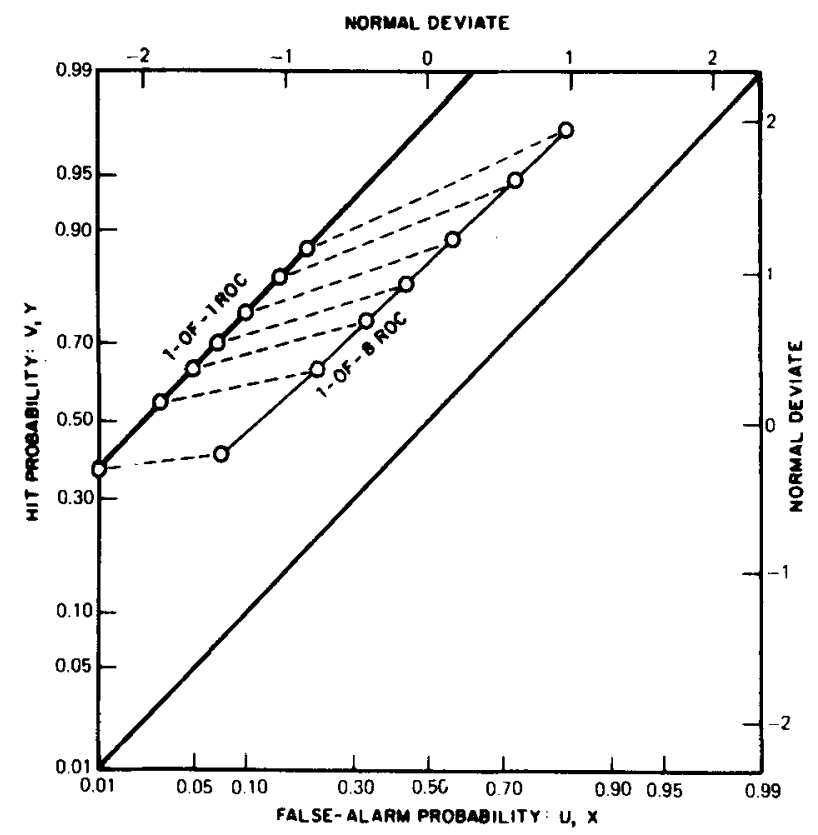

Figure 1. ROC curve for one of eight orthogonal signals relative to the ROC curve for a signal specified exactly, following Nolte and Jaarsma (1967).

Figure 1 gives an example of the effect on performance when the ideal observer is faced with oneof-M orthogonal signals, compared to a signal specified exactly. $M$ here is 8 , eight being the number of frequencies used in our experiment. A value of $\mathrm{d}^{\prime}=2.0$ was chosen for the "one-of-one" ROC curve. The coordinates of points along this curve are denoted $(\mathrm{u}, \mathrm{v})$. Following Nolte and Jaarsma (1967), we obtain the coordinates of corresponding points $(x, y)$ on the "one-of-eight" ROC curve: $\mathrm{x}=1-(1-\mathrm{u})^{8}, \mathrm{y}=1-(1-\mathrm{u})^{7}(1-\mathrm{v})$. These points fall along a nearly normal ROC curve with an index $\mathrm{d}^{\prime}=1.10$ and a slope of about 0.875 . [This detection index, taken at the negative diagonal, is sometimes denoted $d_{e}^{\prime}$ (see Green \& Swets, 1966, p. 97).] It can be seen that the one-of-eight ROC curves are sufficiently straight on a binormal graph (between 0.01 and 0.99) for psychophysical data.

The next step is to establish a relationship between various values of $d^{\prime}$ for SSE and the corresponding values of $d$ ' for one-of-eight frequencies. For this purpose, we took several points along the negative diagonal for the case of one of eight frequencies and solved backwards to find the corresponding locus for the case of a single frequency. That is, in the model for one-of-eight orthogonal signals, eight parallel filters have individual decision criteria; we determined the ROC point for the single filter that passed the signal. The result is shown in Figure 2. The value of $d^{\prime}$ for each point is obtained by subtracting the normal-deviate value on the abscissa from the normal-deviate value on the ordinate.
Now, reading values of $d^{\prime}$ for corresponding points of the two sets and plotting $d^{\prime}(8)$ vs. $d^{\prime}(1)$ leads to the middle curve of Figure 3 . Using this curve, we determine a prediction of $d^{\prime}$ for the composite signal from the value of $d^{\prime}$ obtained under SSE, at each stage of observation. For the simple signal, only the predicted d' for the first observation is determined in this way, and the $\sqrt{\mathbf{n}}$ multiplier is applied to yield predicted values for subsequent observations.

Two other curves are shown in Figure 3. The top curve is $d^{\prime}(8)=d^{\prime}(1)$, which is the result if the ideal observer of the composite signal has available an externally sensed parameter (ESP), i.e., if it has perfect information about the particular value of the random parameter that will occur on any given trial, rather than only information about the distribution of the random parameter (Spooner, 1969). The bottom curve is $d^{\prime}(8)=d^{\prime}(1) / \sqrt{8}$; this curve represents the so-called "multiband" observer, which we describe shortly.

First note that the Nolte-Jaarsma curve for one-ofeight frequencies agrees quite well with the prediction of the multiband model for $\mathrm{d}^{\prime}(1)$ below 1.0 , and that it is roughly $\mathrm{d}^{\prime}(8)=\mathrm{d}^{\prime}(1)-0.90$ for $\mathrm{d}^{\prime}(1)$ between 2.0 and 4.0 .

We may also note that the curves of Figure 3, as it happens, portray qualitatively the growth of detectability over time for the various conditions. That is to say, if we consider Figure 3 to be a plot of $d^{\prime}$ vs. $n$ (number of observations), then the top curve, for the ESP or SSE observer, and the bottom

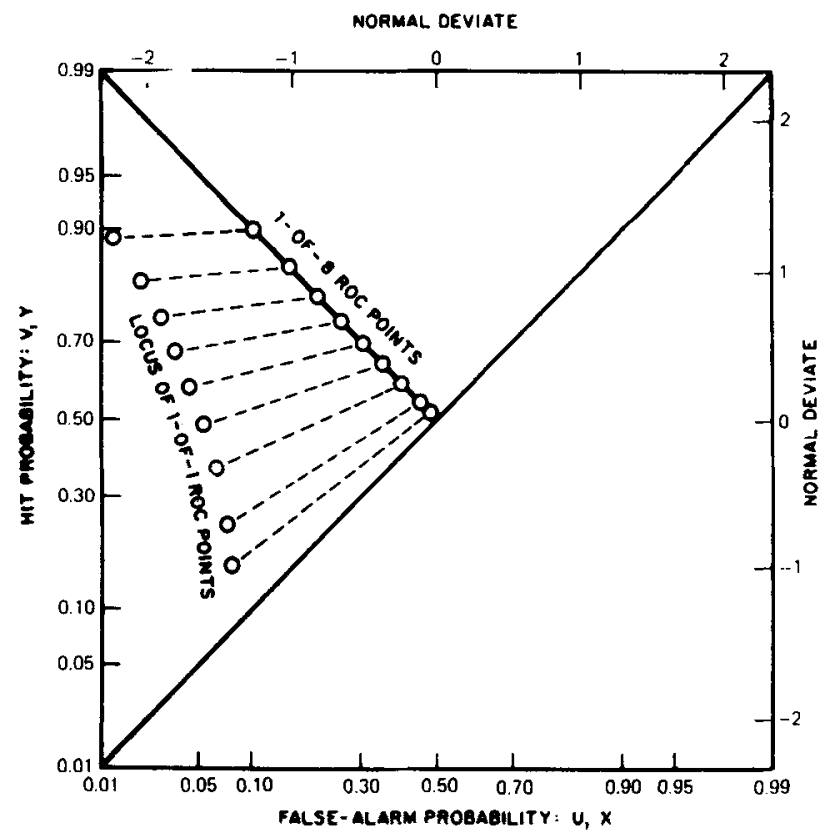

Figure 2. Illustrating the calculation of the relationship of $d^{\prime}$ ' for one-of-eight frequencies to $d$ ' for a single frequency; see text. 


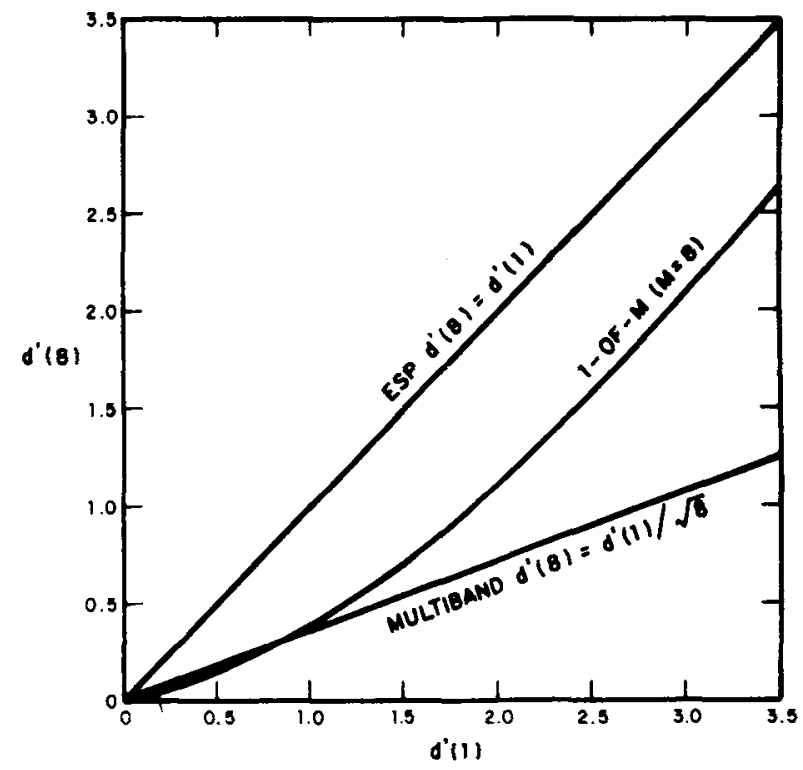

Figure 3. Based on Figure 2, the relationship of $d^{\prime}(8)$ to $d^{\prime}(1)$ predicted by the model for one-of-M orthogonal signals (middle curve); the same relationship for a signal specified exactly (top curve), and for an uncertain signal according to the multiband model (bottom curve).

curve, for the multiband observer, represent the uniform growth of detectability over time that characterizes these observers. On the other hand, the middle curve, for the composite-signal observer, shows a "knee effect," suggesting the ability of that observer to zero in on the signal or to adapt to the uncertain parameter that becomes less uncertain as evidence accumulates in time. Once past the knee, detectability for $\mathbf{M}>1$ rises at the same rate as that for $\mathbf{M}=1$.

\section{The Multiband Observer}

The multiband model, as described in previous literature (see Green \& Swets, 1966, chapter 10), is an alternative to the model for one-of-M orthogonal signals. Briefly, it assumes that on every observation the output of all $M$ bands, or filters, are linearly combined with optimal weighting. If the filters do not overlap and if signals are simultaneously passed by all filters, this model for combining signal frequencies is conceptually identical to the model for combining repeated observations: for equally detectable signals, the predicted $d^{\prime}$ for $M$ frequencies is the $d^{\prime}$ for one frequency multiplied by $\sqrt{M}$. In the present case, with a signal passed by only one filter, but with the noise of all $M$ filters entering the linear combination, the predicted $d^{\prime}$ for one-of-M frequencies is the $\mathrm{d}^{\prime}$ for one frequency divided by $\sqrt{\mathrm{M}}$.

We will use the formula $d^{\prime}(8)=d^{\prime}(1) / \sqrt{8}$ to establish the predicted value of $d^{\prime}(8)$ on the first observation of a trial, and, assuming statistical independence of observations over time, extend the pre- diction to subsequent observations by multiplying by $\sqrt{n}$. As mentioned, the multiband model is an alternative to ones described above for both simple and composite signals. That is, it describes an alternative way of accounting for the effect of frequency uncertainty, and it describes an alternative mode of storing information and of accumulating this information over time. Of course, when extended from the first observation to subsequent observations by $\sqrt{n}$, this model is not an ideal model for the composite signal.

\section{EXPERIMENT}

\section{Method}

The experiment was controlled, and the signals were generated, by a PDP- 8 computer. The duration of the signals was $0.1 \mathrm{sec}$. They were presented through earphones in a continuous background of noise, which had a spectrum level for all observers of approximately $50 \mathrm{~dB}$ SPL. Both signals and noise were presented binaurally.

The eight signal frequencies and their respective values of $E / N_{0}$ were: $490,14.5 ; 675,12.9 ; 880,15.2 ; 1,170,14.2 ; 1,550,16.5$; $2,175,19.2 ; 2,725,19.2 ; 3,425,21.3$. These values of $E / N_{0}$ were selected on the basis of the first 15 daily sessions to yield approximately equal detectability of each frequency; the next three sessions confirmed the approximate equality.

The observers were three female high-school seniors. They observed $2 \mathrm{~h}$ a day, 5 days a week, for approximately 10 weeks.

Trials with the signal specified exactly contained six observation intervals; trials with the simple and composite signals contained 12 observation intervals. Each observation interval (of $0.1 \mathrm{sec}$ ) was preceded by a warning light (of $0.3 \mathrm{sec}$ ) and a space (of $0.5 \mathrm{sec}$ ), and was followed by a response period (of $1.5 \mathrm{sec}$ ). A "yes" or "no" response was made by pushing one of two buttons. Following the last response of a trial, a $(0.5 \mathrm{sec})$ light indicated whether or not a signal had been presented on that trial. The time between trials was $0.5 \mathrm{sec}$. The probability of signal occurrence on a trial was always 0.5 . When simple and composite signals were presented, the probability of each of the eight signal frequencies was $1 / 8$. The observers were informed of the type of listening task before each change of task.

After the first 18 sessions of practice on the eight frequencies with only one observation interval per trial, 10 sessions with repeated observations were devoted to SSE. In each session, a group of 25 trials was devoted to each of the eight frequencies. The first 2 of the 10 sessions were considered practice. Combining the remaining 8 sessions provided 200 trials (each with six observations) at each signal frequency.

The next 10 sessions alternated groups of 12 trials each between the simple and the composite signals. Four groups of trials were devoted to each type of signal in each session. Again, the first 2 of these 10 sessions were considered practice. Combining the remaining 8 sessions provided 384 trials (each with 12 observations) for each type of signal.

The next four sessions were replications of the earlier sessions with SSE, and the concluding four sessions were replications of the earlier sessions with simple and composite signals. In all, then, there were 300 trials per frequency under SSE, which were combined to give a total of 2,400 trials for the SSE data; data on the simple signal are based on 576 trials; and data on the composite signal are based on 576 trials.

\section{Results}

Static relationship. Consider first the relationship between performance with one signal and perform- 

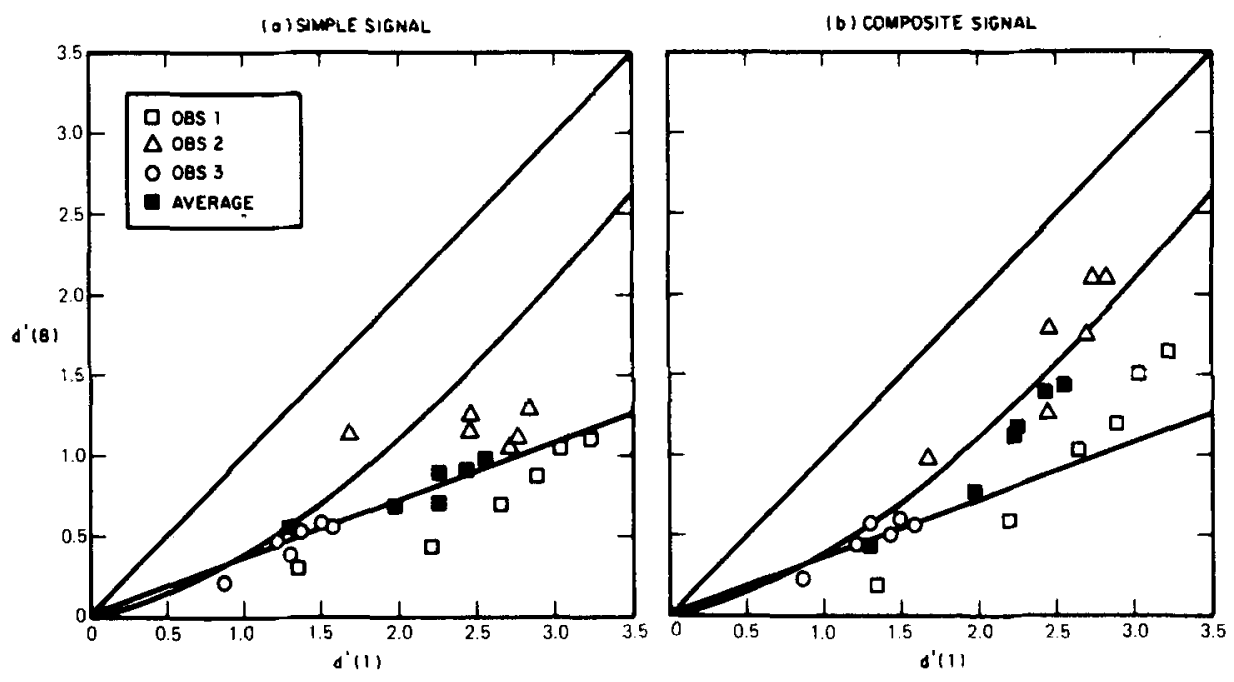

Figure 4. Static analysis of the effect of frequency uncertainty on human performance, relative to the models summarized in Figure 3: (a) simple signal, (b) composite signal.

ance with one-of-eight signals, the latter in both the simple-signal and composite-signal conditions, without regard to the temporal development of performance. That is to say, consider values of $d^{\prime}$ derived from each of the first six observation stages, in all three conditions, on the coordinates of Figure 3 and with respect to the models represented in that figure. Such a comparison acknowledges, of course, that the derivations from $d^{\prime}(1)$ do not depend on how the observer arrives at a given $\mathrm{d}^{\prime}(1)$, whether by variation in number of observations or signal-to-noise ratio.

Figure 4a shows the data from the three observers individually, and the average result, for the simple signal. The data points of Observer 1 fall consistently below the multiband prediction, but generally follow the form of that prediction. Observer 2 does better than the multiband observer, but not as well as the ideal one-of-M observer. The signal levels chosen led to values of $d^{\prime}(1)$ for Observer 3 that were too low to permit a distinction between the one-of-M and multiband models, but Observer 3 also is clearly not an ESP observer.

The average of the three observers closely follows the multiband prediction. We do not necessarily conclude that the multiband model describes the "typical human observer" of the simple signal, but we note for comparative purposes that the simple-signal data cluster about the multiband prediction.

Figure $4 \mathrm{~b}$ shows data obtained with the composite signal. Observer 1 falls consistently below the ideal one-of-M prediction, but generally follows the form of that prediction. Observer 2 also follows the one-of-M prediction, and, if anything, she exceeds it. (Performance superior to the ideal one-of-M prediction is consistent with a somewhat depressed SSE per- formance, and we shall see some evidence for such SSE performance shortly.) Again, Observer 3's relative insensitivity precludes contrasting the one-of-M and multiband models, and is consistent with both.

The average of the three observers adheres fairly closely to the one-of-M prediction. Again, we do not claim the average result to be that of the typical human observer, but we note that composite-signal performance generally exceeds simple-signal performance. The indication is that the human observer tunes to the signal frequency. A static analysis of our data is thus consistent with an attentional process in auditory detection (Green \& Swets, 1966, chapter 10; Swets, 1963) and with an adaptive process.

Dynamic relationship. The remainder of our analysis concerns the growth in detectability over time as a function of signal uncertainty. Specifically, we consider the simple-signal and composite-signal data relative to various predictions based on SSE data.

Data and predictions are shown in Figure 5 (on double logarithmic scales) for the three observers along with the average. The six squares at the top of each panel represent SSE performance. The straight line in their vicinity has a slope of one-half, and represents the prediction that the SSE d' will grow as the $\sqrt{n}$. Note that the theoretical line is forced through the data point at $n=1$. Generally, the empirical increase in $d^{\prime}$ is close to the $\sqrt{n}$ prediction, or perhaps a little greater, from $n=1$ to $\mathrm{n}=2$, and somewhat less than the prediction from $n=2$ on. (There is a suggestion that Observer 2's SSE performance is depressed at later stages by virtue of approaching a practical ceiling.)

The triangles and circles represent the composite and simple signals, respectively. We may note first that both of these types of signal yield performance 


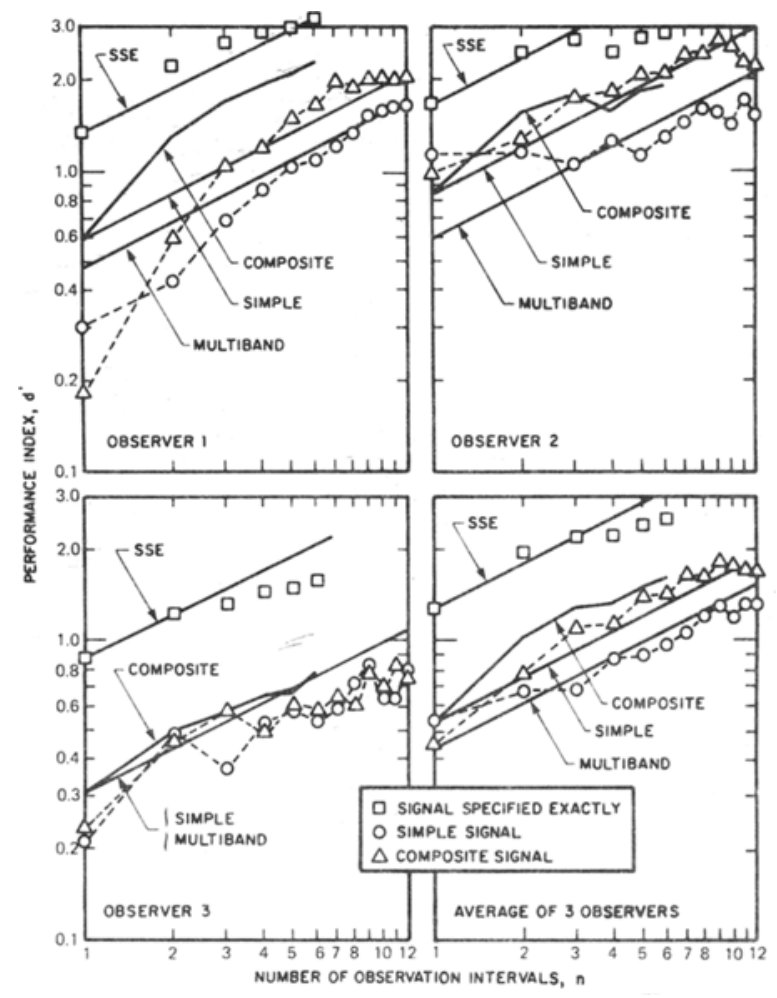

Figure 5. Human detection performance as a function of the number of observations, for three signal conditions, relative to four models.

inferior to the SSE, because of the increase in uncertainty. Considering the average result, the decrement in $d^{\prime}$ is about a factor of 3 . Since $d^{\prime}$ is approximately proportional to signal power (Green \& Swets, 1966, chapter 7), this decrement amounts to about $5 \mathrm{~dB}$. Measuring the effect of uncertainty in the horizontal direction, that is, in terms of time, shows a decrement of about five observations.

Secondly, Observers 1 and 2 perform better on the composite signal than on the simple signal; Observer 3 , perhaps because all the signals were rather weak for her, shows no difference.

Concerning specific predictions, let us look first at composite-signal performance. Observer 1 yields a composite-signal performance below the ideal composite-signal prediction, and, for later observations, above the simple-signal and multiband predictions. The phenomenon we have described as adaptation-zeroing in on, or tuning to, the signal frequency in early observations-is pronounced for Observer 1. The knee effect described in connection with Figure 3 translates on double logarithmic scales into a curve like that followed by Observer 1's composite-signal data: a steep rise at first, followed by a slope approximating the SSE slope.

Observer 2's composite-signal data are not far off the composite-signal prediction. We have noted before the possibility that depressed SSE perform- ance (at later stages) permits composite-signal performance equal to, or surpassing, the prediction defined as ideal. Her composite-signal data generally exceed the simple-signal and multiband predictions. Observer 3's multiple-signal data fall short of all three predictions.

Consider now simple-signal performance. For Observer 1, the slope of the points at later stages is similar to the slope of the simple-signal and multiband prediction, and the level of the points is nearest the multiband prediction. The same is true for Observer 2, though for her the first few points (on the left) are higher rather than lower (indeed, her simple-signal data are almost flat). Observer 3's slope is like the slope of both relevant predictions, and her level falls a little short of both.

Overall, the average result is relatively memorable. The SSE $d^{\prime}$ grows at a rate a little less than $\sqrt{n}$. Simple-signal performance is close to the multiband prediction and consistently below (with the same slope as) the simple-signal prediction. Compositesignal performance exceeds both of the predictions based on no adaptation, and is generally within $1 \mathrm{~dB}$ of the composite-signal prediction.

\section{CONCLUSIONS}

The data are consistent with the following general propositions: the human observer (1) can accumulate sensory information over time in a near optimal manner; (2) suffers a decrement in detection performance from signal uncertainty that is reliable and substantial; (3) can adaptively adjust sensory apparatus to reflect changing knowledge of the stimulusattending to a wide range of frequencies or to a narrow range, as appropriate. The models developed to study these perceptual effects supply heuristic definitions of underlying processes, and yield quantitative results that match up reasonably well with several aspects of human performance.

\section{REFERENCES}

Green, D. M., \& Swets, J. A. Signal detection theory and psychophysics. New York: Wiley, 1966. (Reprinted by Krieger, Huntington, New York, 1974.)

Nolte, L. W. Theory of signal detectability: Adaptive optimum receiver design. Journal of the Acoustical Society of America, 1967, 42, 773-777.

Nolte, L. W., \& JaARsma, D. More on the detection of one of $M$ orthogonal signals. Journal of the Acoustical Society of America, 1967, 41, 497-505.

SPOONER, R. L. Comparison of receiver performance: The ESP receiver. Journal of the Acoustical Society of America, 1969, 45, 233-236.

SwETs, J. A. Central factors in auditory frequency selectivity. Psychological Bulletin, 1963, 60, 429-440.

Swets, J. A., Green, D. M., Getty, D. J., \& Swets, J. B. Signal detection and identification at successive stages of observation. Perception \& Psychophysics, 1978, 23, 275-289.

(Received for publication August 29, 1977; revision accepted February 14, 1978.) 